\title{
Implementación del aprendizaje basado en problemas (ABP) y el learning by doing en el Grado en Publicidad y Relaciones Públicas para la adquisición de competencias
}

\author{
Carmen Llorente BARRoso \\ Universidad CEU San Pablo (España) \\ carmen.llorentebarroso@ceu.es \\ Ángel Bartolomé MuÑoz DE LunA \\ Universidad CEU San Pablo (España) \\ abartolome@ceu.es \\ Mónica VIÑARÁs ABAD \\ Universidad CEU San Pablo (España) \\ monica.vinarasabad@ceu.es
}

\begin{abstract}
Resumen
El marco del EEES (Espacio Europeo de Educación Superior) ha previsto un acercamiento entre el ámbito universitario y el empresarial con el objetivo de optimizar el aprendizaje del alumno y facilitar su entrada en el mundo profesional. Este trabajo resume la experiencia que, con tal propósito, profesores en el Grado de Publicidad y Relaciones Públicas de la Universidad CEU San Pablo hemos diseñado y llevado a cabo con el "Concurso Emprendedores y Creativos", una metodología de innovación docente que combina el ABP (aprendizaje basado en problemas) bajo el método learning by doing.
\end{abstract}

Palabras clave: Espacio Europeo de Educación Superior (EEES), innovación docente, aprendizaje basado en problemas (ABP), learning by doing

Deploy problem based learning (PBL) and learning by doing in the Degree in Advertising and Public Relations for the acquisition of skills

\begin{abstract}
The EHEA (European Higher Education) framework has foreseen an approach between University and business in order to optimize student learning and facilitate their entry into the professional world. With this purpose, lecturers in the Degree in Advertising and Public Relations at San Pablo CEU University have designed and carried out the "Contest Entrepreneurs and Creatives". It consists of an innovative teaching methodology that combines PBL (problem based learning) with learning by doing.
\end{abstract}

Key Words: European Higher Education Area (EHEA), teaching innovation, problem-based learning (PBL), learning by doing

Referencia normalizada:

Llorente Barroso, C.; Bartolomé Muñoz de Luna, A. y Viñarás Abad, M. (2013) Implementación del aprendizaje basado en problemas (ABP) y el learning by doing en el Grado en Publicidad y Relaciones 
Públicas para la adquisición de competencias. Historia y Comunicación Social. Vol. 18. № Especial Noviembre. Págs. 639-650.

Sumario: 1. Introducción. 2. Metodología. 3. El "Concurso Emprendedores y Creativos". 3.1. Ejecución de la metodología. 3.2. Valoración de la metodología. 4. Conclusiones. 5. Referencias bibliográficas

\section{Introducción}

La adaptación de la normativa establecida por el EEES al Grado en Publicidad y Relaciones Públicas requiere el planteamiento de metodologías docentes que impliquen varias disciplinas de forma conjunta. Este es el único camino para lograr que el alumno se enfrente a las dificultades que supone un briefing real y las solucione. Sin embargo, la interdisciplinaridad debe combinarse con otras estrategias metodológicas que permitan al alumno adquirir las competencias profesionales con eficacia. El ABP y el learning by doing pueden considerarse dos de estas estrategias. Se trata de métodos para los que la adquisición de conocimientos de forma proactiva es una parte más del proceso de formación del alumno (Calvo Bernardino \& Mingorance Arnáiz, 2009). La aplicación de estas fórmulas que consideran al alumno el responsable máximo de su propio aprendizaje (Ortiz Sobrino, 2009), potencia las identidades profesionales de los estudiantes, lo que favorece su porvenir profesional (Aldas et al., 2010).

El learning by doing nace en 1999 respondiendo a la exigencia que nos impone nuestra propia vida de "hacer" más que "saber" (Schank, Berman \& Macpherson, 1999). Desde el paradigma cognitivo se ha probado que el "verdadero conocimiento" se adquiere al ejecutar un rol activo, es decir, cuando practicamos, nos equivocamos y corregimos nuestros errores (Martínez Aldanondo, 2003). En este sentido, el aprendizaje busca la "construcción activa de conocimiento", por lo que no puede interpretarse como un proceso en el que un profesor "suministra información" a un alumno que la consume (Martínez Aldanondo, 2006: 37). El alumno tiene que practicar para poder asentar los conocimientos y aplicarlos en su vida. Esta es la finalidad del "Concurso Emprendedores y Creativos", que pretende que el alumno resuelva un problema real orientado por sus profesores.

El ABP propuesto por Barrows (1986) es un método de aprendizaje basado en el constructivismo, que consiste en utilizar problemas para adquirir conocimientos. Se caracteriza tanto por garantizar la adquisición de conocimientos como por facilitar la consecución de habilidades y el desarrollo de actitudes importantes para el aprendizaje, como la evaluación crítica, la responsabilidad o las relaciones interpersonales. El ABP tiene sus raíces en dos paradigmas de aprendizaje contextualizado, el aprendizaje cognitivo y la instrucción anclada, que según Morales Bueno y Landa Fitzgerald (2004), destacan la importancia de contextualizar la enseñanza en problemas reales que plantea el mundo profesional. Este método hace posible el aprendizaje de principios aplicables a diversas problemáticas, mediante la exploración de muy diferentes problemas (Branda, 2001). En este sentido, el "Concurso Emprendedores 
y Creativos" propone a los alumnos el problema comunicativo de un anunciante real, algo que les obliga a estudiar e interpretar datos relativos a tal problema, procedentes de fuentes secundarias. Este análisis facilita a los estudiantes una solución original y eficaz al problema propuesto, pero también les ofrece un cúmulo de normas aplicables a situaciones reales similares en su futuro profesional. Además, la participación de una empresa real en la valoración del trabajo del estudiante permite un mayor acercamiento de su aprendizaje a la realidad profesional.

Morales Bueno y Landa Fitzgerald (2004) establecen tres principios relacionados con los procesos cognitivos que rigen el ABP: a) el aprendizaje no es un proceso receptivo, sino constructivo, b) el proceso de metacognición afecta al aprendizaje y al uso que se hace del conocimiento y c) el aprendizaje está determinado por variables contextuales y sociales. En este sentido, el "Concurso Emprendedores y Creativos" satisface estas máximas:

a) En lo que se refiere al primer principio, que establece que el aprendizaje es un proceso de tipo constructivo, es preciso tener en cuenta la estructura asociativa de la memoria y su influencia sobre el aprendizaje. En este sentido, esta propuesta obliga al alumno a reflexionar asociando conceptos que ha adquirido a través de diferentes disciplinas segregadas en el Grado de Publicidad y Relaciones Públicas.

b) En lo que respecta al segundo principio, relativo a la importancia del proceso de metacognición en el aprendizaje y el uso que se hace del conocimiento, es necesario considerar que las capacidades metacognitivas comprenden la capacidad de analizar la conducta de aprendizaje en sí. En este sentido, el proyecto exige al estudiante que razone las diferentes decisiones que toma en la resolución del problema, lo que le empuja a la reflexión sobre las mismas y, por lo tanto, sobre su propio aprendizaje. El alumno exigente se convierte, así, en un aprendiz-experto, capaz de valorar la complejidad de los problemas y su propia evolución en el proceso para solucionarlos.

c) Por último, en cuanto al tercer principio, que establece la influencia que tienen los factores contextuales y sociales en el aprendizaje, este proyecto prevé la importancia del factor social, al obligar al alumno a trabajar en equipo, exponiéndole a las críticas de su grupo para estimular y mejorar su aprendizaje. Por otro lado, el proyecto considera la importancia del factor contextual, ya que el problema concreto de un anunciante real contextualizado en la situación española actual, obliga a los estudiantes a reflexionar y escoger soluciones adecuadas a las necesidades impuestas por las variables contextuales del anunciante y del público objetivo.

El "Concurso Emprendedores y Creativos" es una metodología que plantea y organiza los diversos procesos de aprendizaje del alumno a partir de la formulación de un problema real. Se trata de un modelo que busca como resultado un trabajo realizado por un conjunto de alumnos y profesores implicados con el fin de facilitar la adquisición de competencias esenciales en el ámbito de la Publicidad y las Relaciones Públicas. Una de las grandes ventajas de esta metodología que apuntan las investigaciones psicológicas más novedosas, es que garantiza un aprendizaje más efectivo que el tradicional al considerar la teoría de las inteligencias múltiples (Redkar, 2012). 


\section{Metodología}

En relación con el acercamiento entre Universidad y empresa que plantea el EEES, el objetivo que orienta este modelo de innovación docente es poner en práctica los conocimientos teóricos adquiridos en diferentes asignaturas del Grado en Publicidad y Relaciones Públicas. De tal modo que la hipótesis que se baraja para alcanzar este objetivo es que la implementación del ABP y el learning by doing en la metodología docente utilizada en el Grado en Publicidad y Relaciones Públicas garantiza al alumno la adquisición eficaz de competencias profesionales básicas.

Esquemáticamente, el modelo metodológico del "Concurso Emprendedores y Creativos" consta de tres claves esenciales:

a) El ABP. Los alumnos deben hacer frente a un problema comunicativo planteado por un anunciante real en un briefing, de modo que tienen que proponer una solución profesional al mismo.

b) Learning by doing. Mediante el planteamiento de este problema, se propone a los estudiantes un aprendizaje a través de la aplicación práctica de los conocimientos que adquieren en las clases regladas. El desarrollo de esta propuesta implica la consideración de los siguientes aspectos:

- Se trata de un trabajo en equipo. Los propios alumnos conforman sus respectivos equipos bajo la condición de que el número de integrantes sea entre cuatro y seis.

- Se exige eficacia. Los alumnos tienen que ofrecer una solución viable al problema, estando obligados a investigar y a considerar los datos reales que observen.

Se trata de una competición. Los estudiantes se enfrentan en un concurso similar a los que se producen en el panorama publicitario. Por ello, el matiz competitivo de este trabajo potencia el realismo de este proyecto. En gran medida, el formato de concurso que se plantea está determinado por la Teoría de los Juegos (Von Neumann \& Morgensten, 1944), según la cual, en los juegos de suma cero hay un único ganador. Esta situación de ganador exclusivo es la que se impone en el mercado publicitario y la que se propone en este caso; en ambas situaciones, se "lucha" por la excelencia para conseguir la cuenta (cliente).

c) Evaluación. En el proyecto se integran diversas formas de evaluación con rasgos innovadores:

- Evaluación formativa. Se realiza durante todo el proyecto.

- Evaluaciones finales. Se ejecutan dos evaluaciones finales. La primera, realizada por los profesores que participan en el proyecto y ciertos representantes del anunciante, que elaboran una lista corta con las propuestas más destacadas por su calidad. La segunda, realizada por un jurado profesional a los trabajos seleccionados (lista corta), que decide el ganador del concurso según los criterios de originalidad, eficacia y calidad. Ambas formas de evaluación final 
implican al anunciante, acercando al discente a las complicaciones habituales de los concursos reales en los que las agencias compiten por una cuenta. Además, la participación de los profesionales asegura la implicación del mundo profesional en la evaluación de los trabajos de los estudiantes y, con ello, en la adquisición de sus competencias.

- Evaluación de los alumnos. Los estudiantes valoran la metodología docente, su adecuación y su eficacia para la adquisición de aquellas competencias establecidas por guía docente. Este sistema de evaluación hace posible la retroalimentación del proyecto y su optimización.

\section{El "Concurso Emprendedores y Creativos"}

\subsection{Ejecución de la metodología}

La metodología docente en la que se apoya el "Concurso Emprendedores y Creativos" requiere de la cooperación de un anunciante que plantee un problema de comunicación a los alumnos a través de un briefing. En este documento de estilo profesional, el anunciante expone los objetivos y normas que deben seguir los alumnos para solucionar mencionado problema. Así, la campaña que los discentes propongan como solución debe adecuarse a las condiciones que establece el anunciante en el briefing, relacionadas con su estado en el mercado, sus políticas formales y funcionales y su target. En consecuencia, es el anunciante el que fija las pautas del ABP y el learning by doing, trasladando al espacio académico una problemática real y cotidiana en el mercado publicitario. Esta incipiente etapa del proyecto, tiene lugar al poco tiempo de empezar el semestre académico, teniendo en cuenta que los alumnos que participan en el proyecto de forma más determinante, al cursar tercero de Grado en Publicidad y Relaciones Públicas, ya disponen de conocimientos suficientes para comenzar el trabajo.

Los estudiantes realizan este trabajo desde diferentes materias del área de creatividad publicitaria, si bien, el desarrollo apropiado del mismo les exige conocimientos que adquieren a través de otras asignaturas relacionadas con el análisis del lenguaje publicitario, la psicología del consumidor y las estrategias en comunicación. De este modo, los alumnos utilizarán, en la realización de su proyecto, conceptos impartidos en segundo curso, en las asignaturas de Fundamentos de la Publicidad y Pensamiento Creativo. En tercer curso, que es cuando hacen el trabajo, adquieren conocimientos desde las disciplinas de Dirección de Arte y Producción Publicitaria, las más determinantes para la ejecución de este proyecto. Finalmente, en cuarto curso, los estudiantes asentarán, mejorarán y completarán los conceptos y competencias adquiridas a través de este trabajo, desde las asignaturas de Comunicación Integrada y Comunicación Estratégica. En este sentido, pese a que el ABP que se propone se ejecute concreta- 
mente en tercero de Publicidad y Relaciones Públicas, se integra en una formación a lo largo de todo el Grado, lo que posibilita dos evaluaciones de interés:

a) La de los docentes que participan en el proyecto, que pueden corroborar el progreso en el aprendizaje de sus alumnos a lo largo del Grado.

b) La del propio alumno, que puede analizar la progresión de su madurez y excelencia a la hora de afrontar las situaciones problemáticas que se le plantean a lo largo del Grado.

El ABP de esta propuesta se apoya en las pautas del learning by doing, con el objetivo de potenciar la capacidad del alumno para estructurar y planificar proyectos complejos en equipo, desde su puesta en práctica. Esto significa, que se considera imprescindible desarrollar la solución al problema, dado que "practicar" constituye la mejor fórmula para adquirir ciertas competencias publicitarias. Esta estrategia de "aprender haciendo" precisa utilizar algunas herramientas que aseguren su eficacia:

a) La pre-evaluación. Este instrumento permite averiguar el nivel de formación de los alumnos y, consecuentemente, ofrece la posibilidad de descubrir qué alumnos necesitan refuerzo y en qué materias. La pre-evaluación se efectúa al comienzo del proyecto.

b) Las lecciones magistrales. El replanteamiento de las clases magistrales a consecuencia de la irrupción de las Tecnologías de la Información y la Comunicación y de su cuestionada eficacia pedagógica en materias de alta tecnificación (Evans \& Matthew, 2012), ha llevado a plantear lecciones magistrales que integran los conceptos teóricos en análisis de casos (campañas de referencia). Así, se pretende garantizar un mejor y más eficaz aprendizaje de los conceptos teóricos para que los alumnos puedan llevarlos a la práctica con mayor facilidad. Estas lecciones magistrales son especialmente importantes para los alumnos de segundo curso, ya que ofrecen "saberes" en los que se apoya el desarrollo práctico de su carrera profesional.

c) Los talleres-seminarios. Son particularmente relevantes para los estudiantes de tercero, ya que conforman una herramienta básica para aprender conocimientos esenciales de aplicación práctica. Este tipo de clases se organizan para grupos de 15-20 alumnos, que hagan posible una dinámica positiva de trabajo entre profesor-alumno y alumno-alumno. En ellos, los estudiantes adquieren habilidades técnicas y destrezas expresivas propias del lenguaje publicitario para poder ejecutar los conceptos creativos con alto nivel de calidad. Este tipo de talleres se desarrollan en aulas equipadas para la adquisición de conocimientos prácticos mediante learning by doing.

d) Los talleres-tutorías. Son talleres estructurados para grupos de alumnos muy reducidos (de cuatro a seis), planificados exclusivamente en las disciplinas de Dirección de Arte y Producción Publicitaria. Los profesores de mencionadas asignaturas son los encargados de tutorizar los proyectos de los diferentes grupos, supervisándoles en las dificultades que deben superar (ABP) mediante un sistema de evaluación continua y formativa. Cada dos semanas, los grupos deben acudir a estas tutorías para que el docente pueda hacer un seguimiento de sus respectivos trabajos, examinando 
junto a ellos las posibles alternativas para optimizar sus proyectos respecto a las necesidades que plantea el briefing. De este modo, se genera un feedback formativo que permite a los estudiantes mejorar sus destrezas y conocimientos.

e) El informe de resultados entre docentes de distintas disciplinas. La colaboración entre profesores es indispensable. El rasgo interdisciplinar del "Concurso Emprendedores y Creativos" precisa una transferencia informativa entre los docentes de las disciplinas más implicadas en el proyecto (Dirección de Arte y Producción Publicitaria), dado que se cursan en semestres diferentes. En el informe de resultados, el profesor de Dirección de Arte (impartida en el primer semestre) transmite al profesor de Producción Publicitaria (impartida en el segundo semestre) una evaluación de cada grupo de trabajo especificando los objetivos logrados, las competencias adquiridas y las alternativas de optimización de los trabajos. De esta manera, tal informe conforma, en el segundo semestre, la pre-evaluación que se hace en el primer semestre.

f) El uso de las Tecnologías de la Información y la Comunicación. Se ha demostrado que los entornos digitales aplacan la timidez que suele dificultar la participación de los alumnos en las aulas (Evans \& Matthew, 2012). Por este motivo se han utilizado las nuevas tecnologías en la realización de este proyecto, tratando de motivar la interacción profesor-alumno y facilitando una nueva vía de acceso a los materiales necesarios para la ejecución del trabajo.

g) Los sistemas para evaluar los trabajos de los alumnos. Se combinan tres formas de evaluación:

- Un feedback formativo. Se trata de una evaluación continua que busca mejorar los trabajos de los alumnos, consolidar sus conocimientos y pulir sus competencias.

- Una pre-evaluación final, criterial y normativa. Se trata de una evaluación final a la que se someten los trabajos de todos los grupos. Los alumnos deben exponer sus trabajos en una sesión abierta, para ello cuentan con siete minutos en los que tienen que presentar un resumen estructurado de su trabajo a un jurado formado por los profesores que participan en el proyecto y por representantes del anunciante. Al tratarse de una sesión abierta, todos los participantes en el "Concurso Emprendedores y Creativos" tienen la posibilidad de presenciar las presentaciones de sus compañeros, de modo que tal sesión adquiere un matiz formativo. De esta sesión se extrae una lista corta de finalistas, constituida por los cinco mejores trabajos y confeccionada por el jurado según los criterios de originalidad, eficacia y calidad. Esta lista es la que se someterá a la evaluación final.

- Una evaluación final, criterial y normativa. Únicamente se someten a esta evaluación los trabajos elegidos como finalistas, si bien todos los estudiantes que participan en el proyecto están convocados a esta sesión, ya que la lista corta no se hace pública hasta este momento. Así, se quiere que el discente 
lleve a práctica su habilidad para controlar el factor sorpresa y reaccionar con rapidez y eficacia. Esta valoración final la efectúa un jurado formado por representantes del anunciante, autoridades académicas de la Universidad CEU San Pablo y reconocidos profesionales del mundo de la publicidad. El resultado de la resolución de este jurado decide el vencedor del "Concurso Emprendedores y Creativos". Esta jornada, programada al final del curso académico, pretende ser un reconocimiento a los alumnos que han participado en el proyecto.

\subsection{Valoración de la metodología}

La evaluación de la metodología docente que se propone, la realizan sus receptores-consumidores, es decir, los alumnos. Para ello, los estudiantes realizan una encuesta con la que se pretende conocer su grado de satisfacción con la metodología y la medida en la que les ha permitido adquirir competencias. Mencionada encuesta está construida de acuerdo a las metas establecidas por guía docente para cada asignatura implicada en el proyecto. Esta encuesta la realizan todos los alumnos que han participado en "Concurso Emprendedores y Creativos" de forma individual y anónima, durante la jornada de pre-evaluación final.

La Tabla 1 muestra los resultados más sobresalientes, referidos a la evaluación de la metodología de innovación docente que se ha explicado anteriormente y se efectuó durante el curso 2011-2012.

Tabla 1. Evaluación de la metodología docente.

\section{La participación en el "Concurso Emprendedores y Creativos" permite...}

\begin{tabular}{|l|c|c|c|c|c|}
\hline & $\begin{array}{c}\text { Totalmente } \\
\text { insatisfecho }\end{array}$ & Insatisfecho & Indiferente & Satisfecho & $\begin{array}{c}\text { Muy } \\
\text { satisfecho }\end{array}$ \\
\hline $\begin{array}{l}\text { 1. Una mejor interpretación del } \\
\text { briefing de un anunciante real }\end{array}$ & 2,9 & 5,4 & 18,9 & $\mathbf{4 1 , 4}$ & 31,4 \\
\hline $\begin{array}{l}\text { 2. Una adaptación a las necesidades } \\
\text { y pautas del anunciante }\end{array}$ & 2,8 & 2,8 & 18,9 & 35,1 & $\mathbf{4 0 , 4}$ \\
\hline $\begin{array}{l}\text { 3. Una más efectiva interpretación } \\
\text { de los objetivos de la campaña }\end{array}$ & 3,6 & 3,6 & 18,1 & $\mathbf{4 9 , 5}$ & 25,2 \\
\hline $\begin{array}{l}\text { 4. El desarrollo de estrategias } \\
\text { considerando el briefing y otros } \\
\text { datos reales }\end{array}$ & 1,8 & 3,6 & 22,4 & $\mathbf{4 6 , 1}$ & 26,1 \\
\hline $\begin{array}{l}\text { 5. La consolidación de aptitudes } \\
\text { necesarias para afrontar el mercado } \\
\text { laboral }\end{array}$ & 2,8 & 4,5 & 14,4 & $\mathbf{4 9 , 5}$ & 28,8 \\
\hline $\begin{array}{l}\text { 6. La ejecución del trabajo de } \\
\text { Grado más próximo a la profesión }\end{array}$ & 3,6 & 2,9 & 15,7 & $\mathbf{3 2 , 4}$ & $\mathbf{4 5 , 4}$ \\
\hline $\begin{array}{l}\text { 7. El uso y la comprensión de } \\
\text { términos del argot profesional que } \\
\text { no conocía }\end{array}$ & 2,9 & 6,3 & 25,3 & $\mathbf{4 5 , 2}$ & 20,3 \\
\hline
\end{tabular}

Fuente: Elaboración propia (muestra de 111 alumnos -todos los participantes en el proyecto-; resultados ponderados sobre el total de esta muestra), 14 de mayo de 2012. 
Un análisis global de los resultados de la encuesta permite determinar un alto nivel de satisfacción por parte de los alumnos respecto a la metodología de innovación docente desarrollada. Los discentes que han participado en el proyecto piensan que se trata de una fórmula que les aproxima al mundo profesional y les ofrece la posibilidad de adquirir competencias básicas para desenvolverse en él. Esta satisfacción abarca aproximadamente al $75 \%$ de los alumnos que han participado en el proyecto, quienes califican con valoraciones de "satisfecho" y/o "muy satisfecho" la mayoría de los items por los que se les pregunta. De este modo, el Gráfico 1 señala hasta un $73,8 \%$ de valoraciones positivas en la media ponderada del total de valoraciones de la metodología docente aplicada.

Gráfico 1. Media ponderada de las valoraciones de la metodología docente aplicada.

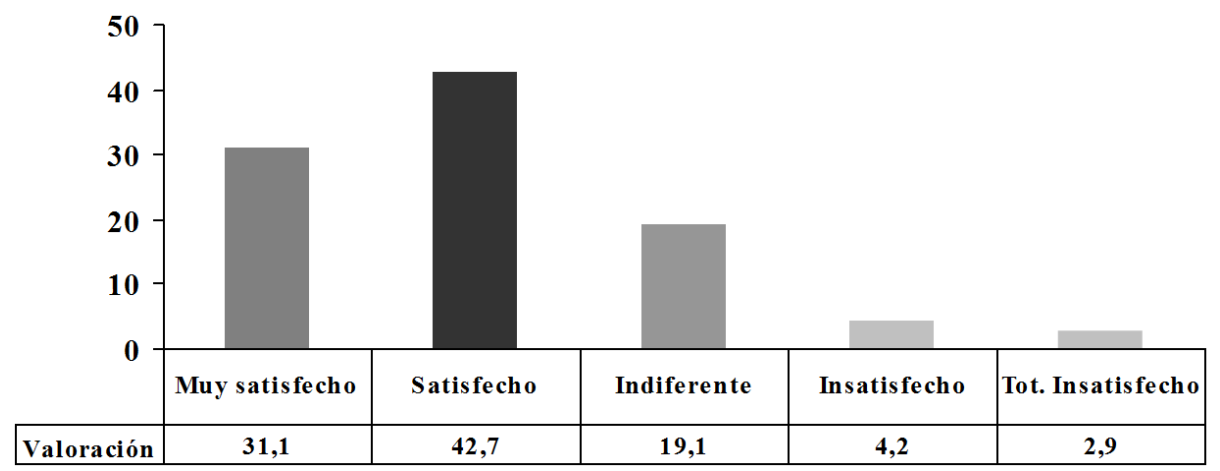

Fuente: Elaboración propia (muestra de 111 alumnos -todos los participantes en el proyecto-; resultados ponderados sobre el total de esta muestra), 14 de mayo de 2012.

Estos resultados permiten a los docentes que han participado en el proyecto:

- Descubrir los aspectos que deben mejorarse en diseño y organización de la metodología de innovación docente para el siguiente curso académico, ya que permite detectar carencias o posibles mejoras.

- En segundo lugar, averiguar las actividades más satisfactorias y efectivas para el aprendizaje del alumno desde su propio punto de vista.

\section{Conclusiones}

El nivel de satisfacción reflejado por los alumnos permite corroborar la hipótesis de partida, "la implementación del ABP y el learning by doing en la metodología docente utilizada en el Grado en Publicidad y Relaciones Públicas garantiza al alumno la adquisición eficaz de competencias profesionales básicas", ya que le plantea un situación problemática real en su ámbito profesional y le exige una implicación activa para resolverla. 
La metodología que se propone en el "Concurso Emprendedores y Creativos" es acorde a la normativa planteada por el EEES, al fundamentarse en dos métodos (en particular, el ABP y, en concreto, el learning by doing) que convierten al discente en el agente más importante para su aprendizaje y formación, haciendo que se responsabilice y participe activamente en la adquisición y optimización de sus competencias. El ABP propone a los estudiantes el problema de un anunciante que tienen que solucionar. Para ello, están obligados a poner en práctica conocimientos teóricos mediante la fórmula del learning by doing que les lleva a afrontar problemáticas propias de la profesión publicitaria. De este modo, se consigue que el alumno practique con estas situaciones que se encontrará en su experiencia profesional (Martínez Aldanondo, 2006). Este planteamiento metodológico incorpora un sistema de evaluación complejo que posibilita a los profesores, por un lado, el seguimiento de los trabajos durante el curso con el fin de optimizarlos, y por otro lado, la valoración definitiva de los mismos al final del curso. Asimismo, los alumnos realizan una evaluación de la metodología docente, lo que hace posible, en primer lugar, averiguar la eficacia y oportunidad de tal metodología según los propios estudiantes, y en segundo lugar, optimizar el proyecto a partir del feedback de los alumnos.

En el desarrollo de esta metodología, el docente simplemente guía al alumno (Bartolomé Muñoz de Luna, Viñarás Abad \& Llorente Barroso, 2012) con el objetivo final de que logre desarrollar su identidad profesional (Aldas et al., 2010) desde su acercamiento activo a todas las asignaturas que participan en el proyecto.

El "Concurso Emprendedores y Creativos" afronta los retos que suscita el EEES respecto a la formación en competencias particulares. En este sentido, esta metodología está delineada para conseguir que el alumno piense, reflexione, examine y actúe de forma interdisciplinar, y de este modo, adquiriera las destrezas y asuma las responsabilidades propias de su ámbito profesional. Entre las contribuciones más destacadas que esta metodología aporta a los actores principales del EEES, destacan:

a) Al alumno. Los estudiantes consiguen desarrollar una percepción integrada de conocimientos (teóricos y prácticos). Aprenden a analizar su progresión a lo largo del trabajo y del Grado y consiguen trabajar adaptándose a las pautas del contexto empresarial. En este sentido, su formación se desarrolla inmersa en el contexto real de la problemática (Morales Bueno \& Landa Fitzgerald, 2004).

b) Al docente. Los profesores trabajan en colaboración con sus colegas, debiendo unificar criterios y consiguiendo que el aprendizaje de sus alumnos tenga una continuidad. Esta forma de trabajar hace posible una fuerte sinergia entre disciplinas, logrando optimizar su calidad. Asimismo, la interdisciplinaridad ayuda al alumno a comprender con mayor facilidad los diversos aspectos que encierra el problema que deben resolver (Bartolomé Muñoz de Luna, Viñarás Abad \& Llorente Barroso, 2012).

c) A la Universidad. La Universidad genera relaciones de colaboración con el ámbito empresarial, que garantizan al alumno una adquisición más eficaz de sus competencias. Estas propuestas contribuyen también a potenciar la competitividad de la Universidad y su prestigio. 
Por todo ello, el "Concurso Emprendedores y Creativos" se ha consolidado como un método de innovación docente eficaz en la formación de los alumnos de Publicidad y Relaciones Públicas, reconocido por el sector profesional, el mundo académico y los propios alumnos.

\section{Referencias bibliográficas}

ALDAS, T. et al. (2010). "Learning by Doing: The Wagner Plan from Classroom to Career". En: $A A C \& U$. p. 24-28.

BARROWS, H. S. (1986). "A Taxonomy of problem-based learning methods". En: Medical Education, $\mathrm{n}^{\circ}$ 20(6).

BARTOLOMÉ MUÑOZ DE LUNA, Á., VIÑARÁS ABAD, M. \& LLORENTE BARROSO, C. (2012). "Emprendedores y Creativos: una propuesta interdisciplinar de innovación docente". En: Revista de Comunicación Vivat Academia, $\mathrm{n}^{\mathrm{o}}$ 117E. p. 1613-1641. Recuperado [15-10-2013] de http://pendientedemigracion. ucm.es/info/vivataca/numeros/n117E/PDFs/Varios36.pdf

BRANDA, L. (2001). "Aprendizaje Basado en Problemas centrado en el estudiante, orientado a la comunidad". En PULPEIR, A. (dir.) Aportes para un cambio curricular en Argentina 2001. Buenos Aires: Universidad de Buenos Aires. p. 79-101. Recuperado [15-10-2013] de http://www.psico.uniovi.es/fac_psicologia/paginas_eees/Adaptacion_de_profesorado/metodos_docentes/aprendizaje basado_en_problemas_aulafutura.pdf

CALVO BERNARDINO, A. \& MINGORANCE ARNÁIZ, A. C. (2009). "La estrategia de las universidades frente al Espacio Europeo de Educación Superior". En: Revista Complutense de Educación, $\mathrm{n}^{\circ}$ 20(2). Recuperado [15-10-2013] de http:// revistas.ucm.es/index.php/RCED/article/view/RCED0909220319A/15299

EVANS, R., \& MATTHEW, A. (2012). "Should we still lecture? Reconsidering pedagogical approaches to promote student engagement, challenging the traditional lecture". En: INTED 2012, Conference technical program. Recuperado [15-102013] de http://eprints.qut.edu.au/49062/2/49062.pdf

MARTÍNEZ ALDANONDO, J. (2003). "Contenidos en e-learning: El rey sin corona (por ahora)". En www.uoc.edu, FUOC. p. 1-13. Recuperado [15-10-2013] de http://www.uoc.edu/dt/20126/20126.pdf

MARTÍNEZ ALDANONDO, J. (2006). "E-learning en blanco y negro". En: Learning Review, $\mathrm{n}^{\circ}$ 14. p. 36-37. Recuperado [15-10-2013] de http://www.learningreview.com/articulos-y-entrevistas-elearning/360-elearning-en-blanco-y-negro

MORALES BUENO, P. \& LANDA FITZGERALD, V. (2004). “Aprendizaje Basado en Problemas". En: Theoria, no 13(1). Recuperado [15-10-2013] de http://campus. usal.es/ ofeees/NUEVAS METODOLOGIAS/ABP/13.pdf

ORTIZ SOBRINO, M. Á. (2009). "La reconversión de la formación en comunicación desde el espacio europeo de educación superior". En: Revista Icono14, nº 7(3). 
p. 35-49. Recuperado [15-10-2013] de http://www.icono14.net/ojs/index.php/ icono14/article/view/301/178

REDKAR, S. (2012). "Teaching Advanced Vehicle Dynamics Using a Project Based Learning (PBL) Approach". En: Journal of STEM Education, no 13(3). p. 17-29.

SCHANK, R. C., BERMAN, T. R., \& MACPHERSON, K. A. (1999). "Learning by doing", en REIGELUTH, C. M. (Ed.): Instructional design theories and models volume II: A new paradigm of instructional theory. Mahwah, NJ: Lawrence Erlbaum.

VON NEUMANN, J. \& MORGENSTERN, O. (1944). Theory of Games and Economic Behaviour. Princeton: Princenton University Press.

\section{Los autores}

Carmen Llorente Barroso es Doctora en Comunicación Audiovisual y Publicidad por la UCM desde 2010. Ha desarrollado parte de su carrera como investigadora OTRI UCM-ZENITHOPTIMEDIA con cargo a un proyecto de eficacia publicitaria. Miembro activo de R14, CEU-CITEC y ASOCREA, ha publicado un libro y varios artículos en revistas indexadas, participando con cierta regularidad en Congresos nacionales e internacionales. Actualmente, compagina sus labores docentes en la Universidad CEU San Pablo con las investigadoras, centrándose en el estudio de la retórica aplicada a la creatividad publicitaria y en el análisis de indicadores de eficacia comunicativa vinculados al comportamiento del consumidor.

Ángel Bartolomé Muñoz de Luna es Doctor en Comunicación Audiovisual y Publicidad por la Universidad CEU San Pablo. Profesor Adjunto en la Universidad CEU San Pablo y director del Máster Universitario en Diseño Gráfico de la Comunicación en el Instituto de las Artes de la Comunicación TRACOR. Miembro de La Academia de la Publicidad, Icono 14 y ASOCREA. Ha publicado, junto con otros autores, el libro "Los estudios de Comunicación en el EEES" y "La tecnología audiovisual al servicio de la sociedad". Así como varias publicaciones sobre la publicidad y creatividad en revistas especializadas. Recientemente ha realizado una investigación en City London University sobre problem solving y creatividad.

Mónica Viñarás Abad es Doctora en Ciencias de la Información y Licenciada en Publicidad y Relaciones Públicas por la UCM. Profesora Adjunta en la Universidad CEU San Pablo y directora de la Sección de Publicidad y Comunicación Institucional. Forma parte de distintos proyectos de investigación financiados y es miembro de asociaciones profesionales como Icono 14, ASOCREA, AIRP y AE-IC. Ha publicado artículos en diferentes revistas científicas sobre Relaciones Públicas y Comunicación Corporativa, RSC, gestión de la comunicación en instituciones culturales, etc.; en este año 2013 ha coordinado el libro Nuevas Tendencias en la Investigación en Comunicación, de CEU Ediciones. 